\title{
Sticky polymatroids on at most five elements
}

\author{
Laszlo Csirmaz
}

\author{
Dedicated to the memory of Frantisek Matúš
}

\begin{abstract}
The sticky polymatroid conjecture states that any two extensions of the polymatroid have an amalgam if and only if the polymatroid has no non-modular pairs of flats. We show that the conjecture holds for polymatroids on five or less elements.
\end{abstract}

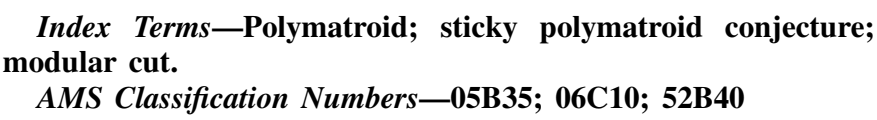

\section{INTRODUCTION}

A polymatroid is sticky if any two of its extensions have an amalgam. This is a direct generalization of the same property of matroids. If every pair of flats is modular then the polymatroid is sticky; the proof in [8] generalizes to polymatroids. The sticky polymatroid conjecture states that the converse also holds: in a sticky polymatroid, each pair of flats is a modular pair. The corresponding conjecture for matroids has been stated by Poljak and Turzik [10], and received a considerable attention. Poljak and Turzik showed that the sticky matroid conjecture holds for rank-3 matroids. Bachem and Kern [1] showed that the same conjecture holds in general if it is true for every rank-4 matroid. Generalizing a result of Bonin in [2] which states that a matroid of rank at least three with two disjoint hyperplanes is not sticky, Hochstättler and Wilhelmi showed that matroids having a nonprincipal modular cut are not sticky [5]. The same statement for polymatroids was proved in [3] using a convolution-type construction. Thus the sticky polymatroid conjecture follows from the statement:

\section{(*) If a polymatroid has a non-modular pair of flats, then it also has a non-principal modular cut.}

In this note we show that $(*)$ holds for polymatroids on ground set with at most five elements. Thus a smallest counterexample to the sticky polymatroid conjecture, if such exists, must have at least six atoms.

Interestingly, the sticky matroid conjecture may not follow from the same conjecture for polymatroids. The reason is that if two matroids have a polymatroid amalgam, the value of the rank function of any subset $A$ of the ground set of either matroid is an integer that is at most $|A|$, the rank of a set that is not a subset of either ground set might not be an integer (but can be rational). Interestingly no such example is known. At the same time, $(*)$, if true, implies the sticky matroid conjecture.

All sets in this paper are finite. Following the usual practice, ground sets and their subsets are denoted by capital letters,

Central European University, Budapest

email:csirmazerenyi.hu their elements by lower case letters. The union sign $\cup$ and the curly brackets around singletons are omitted, thus $a b A$ denotes the set $\{a, b\} \cup A$. The modular defect of subsets $A$ and $B$ is defined as

$$
\delta_{f}(A, B)=f(A)+f(B)-f(A \cup B)-f(A \cap B) .
$$

It is non-negative, and if zero, then the pair $(A, B)$ is called a modular pair.

The paper is organized as follows. Polymatroids, one-point extension, and the excess function are defined in Section III and some basic properties are given. Section III introduces the notion of linear polymatroid. This is an intrinsic property shared by all linearly representable polymatroids. We hope that this notion has further applications. Two lemmas in Section IV describe some properties of a minimal counterexample to $(*)$. Using these lemmas and a property of linear polymatroids, Section $\mathrm{V}$ shows that no polymatroid on five or less elements violates $(*)$.

\section{Definitions}

A polymatroid $\mathcal{M}=(f, M)$ is a real-valued, non-negative, monotone and submodular function $f$ defined on the set of subsets of the finite set $M$ such that $f(\emptyset)=0$. Here $M$ is the ground set, and $f$ is the rank function. The polymatroid is a matroid if all ranks are integers and $f(A) \leq|A|$ for all $A \subseteq$ $M$. For details see [6], [8]. The rank function can be identified with a $\left(2^{|M|}-1\right)$-dimensional real vector, where the indices are the non-empty subsets of $M$. Vectors corresponding to polymatroids on the ground set $M$ form the pointed polyhedral cone $\Gamma_{M}$ [13]. Its facets are the hyperplanes determined by the basic submodular inequalities $\delta_{f}(i K, j K) \geq 0$ with distinct $i, j \in M-K$ and $K \subseteq M$ ( $K$ can be empty), and the monotonicity requirements $f(M) \geq f(M-i)$; see [7, Theorem 2]. Much less is known about the extremal rays of this cone. They have been computed for ground sets up to five elements [12] without indicating any structural property. Fixing a polymatroid on each extremal ray, every polymatroid in $\Gamma_{M}$ is a non-negative linear combination (also called conic combination) of these extremal polymatroids.

\section{A. Flats, modular cuts and filters}

Let $\mathcal{M}=(f, M)$ be a fixed polymatroid. A subset $F \subseteq$ $M$ is a flat if every proper superset of $F$ has strictly larger rank. The closure of $A$, denoted by $\mathrm{cl}(A)$, is the smallest flat containing $A$. The collection $\mathcal{F}$ of flats is a modular cut if it has properties (i)-(iii) below:

(i) closed upwards: if $F \in \mathcal{F}$ and the flat $F^{\prime}$ is a superset of $F$, then $F^{\prime} \in \mathcal{F}$; 
(ii) closed for modular intersection: if $F_{1}, F_{2} \in \mathcal{F}$ and $\left(F_{1}, F_{2}\right)$ is a modular pair (that is, $\left.f\left(F_{1}, F_{2}\right)=0\right)$, then $F_{1} \cap F_{2} \in \mathcal{F}$ (observe that intersection of flats is a flat);

(iii) not empty, which is equivalent to $M \in \mathcal{F}$.

In standard textbooks, such as [8], the empty collection is also considered to be a modular cut. It has been excluded here to emphasize the similarity to modular filters defined below.

The modular cut generated by the flats $F_{1}, \ldots, F_{k}$ is the smallest modular cut containing all of these sets. This modular cut is denoted by $\mathcal{F}\left(F_{1}, \ldots, F_{k}\right)$.

A modular cut $\mathcal{F}$ is principal if it is generated by a single flat; or, equivalently, if the intersection of all elements of $\mathcal{F}$ is also an element of $\mathcal{F}$. When $\mathcal{F}$ is not principal, there are two flats $F_{1}, F_{2} \in \mathcal{F}$ such that $F_{1} \cap F_{2} \notin \mathcal{F}$. In this case $F_{1} \cap F_{2} \notin \mathcal{F}\left(F_{1}, F_{2}\right)$ as the modular cut generated by $F_{1}$ and $F_{2}$ is a subcollection of $\mathcal{F}$.

The collection $\mathcal{G}$ of subsets of $M$ is a modular filter if it satisfies the following properties:

(i) closed upwards: if $A \in \mathcal{G}, A \subseteq B$, then $B \in \mathcal{G}$;

(ii) closed for modular intersection: if $A, B \in \mathcal{G}$ and $(A, B)$ is a modular pair, then $A \cap B \in \mathcal{G}$;

(iii) non-trivial: if $f(X)=f(M)$, then $X \in \mathcal{G}$.

Modular filters generated by certain subsets as well as principal and non-principal modular filters can be defined similarly to modular cuts.

The following Proposition shows how to get a modular filter from a modular cut.

Proposition 1. Suppose $\mathcal{F}$ is a modular cut. The collection $\mathrm{cl}^{-1}(\mathcal{F})=\{A \subseteq M: \mathrm{cl}(A) \in \mathcal{F}\}$ is a modular filter.

Proof. Properties (i) and (iii) of the definition of a modular filter clearly hold, thus suppose $(A, B)$ is a modular pair and both $F_{A}=\operatorname{cl}(A)$ and $F_{B}=\operatorname{cl}(B)$ are in $\mathcal{F}$. Then $\operatorname{cl}\left(F_{A} F_{B}\right)=\operatorname{cl}(A B)$ and $F_{A} \cap F_{B} \supseteq A \cap B$, thus

$$
\begin{aligned}
& f\left(F_{A}, F_{B}\right)=f(A, B)+f(A \cap B)-f\left(F_{A} \cap F_{B}\right) \leq \\
& \quad \leq f(A, B)=0 .
\end{aligned}
$$

Consequently $\left(F_{A}, F_{B}\right)$ is a modular pair and $f(A \cap B)=$ $f\left(F_{A} \cap F_{B}\right)$, thus $\operatorname{cl}(A \cap B)=F_{A} \cap F_{B} \in \mathcal{F}$, and $A \cap B \in$ $\mathrm{Cl}^{-1}(\mathcal{F})$.

\section{B. Extensions}

The polymatroid $\mathcal{M}^{\prime}=\left(f^{\prime}, M^{\prime}\right)$ is an extension of $\mathcal{M}=$ $(f, M)$ if $M^{\prime} \supset M$, and $f(X)=f^{\prime}(X)$ for all $X \subseteq M$. This is a one-point extension if $M^{\prime}-M$ has a single element.

Given a polymatroid $(f, M)$ and extensions $\left(f_{1}, M_{1}\right)$ and $\left(f_{2}, M_{2}\right)$ of $(f, M)$ with $M_{1}-M$ and $M_{2}-M$ disjoint, an amalgam is a polymatroid on $M_{1} \cup M_{2}$ that extends both $\left(f_{1}, M_{1}\right)$ and $\left(f_{2}, M_{2}\right)$. The polymatroid $\mathcal{M}$ is sticky if any two of its extensions have an amalgam.

The function $e$ defined on the set of subsets of $M$ is an $e x$ cess function of $\mathcal{M}=(f, M)$ if there is a one-point extension $\mathcal{M}^{\prime}=\left(f^{\prime}, M \cup\{x\}\right)$ of $\mathcal{M}$ such that $e(A)=f^{\prime}(x A)-f^{\prime}(A)$ for all $A \subseteq M$. If the polymatroid $\mathcal{M}$ is clear from the context, $e$ is called simply an excess function.
Proposition 2. The function $e$ is an excess function if and only if the following conditions hold.

(i) $e$ is non-negative and decreasing: $e(A) \geq e(B) \geq 0$ for $A \subseteq B \subseteq M$,

(ii) $(e(M)-e(M-i))+(f(M)-f(M-i)) \geq 0$ for all $i \in M$,

(iii) $\delta_{e}(a A, b A)+\delta_{f}(a A, b A) \geq 0$ for all $a b A \subseteq M, a, b \notin A$ (including $A=\emptyset$ ).

Proof. It is clear that the conditions are necessary. For sufficiency, it is enough the check that $f^{\prime}$ defined on subsets of $M x$ as $f^{\prime}(A x)=f(A)+e(A)$ and $f^{\prime}(A)=f(A)$ is the rank function of a polymatroid. According to [7, Theorem 2] it is enough to check $f^{\prime}(M) \geq f^{\prime}(M-i)$ for $i \in M x$ and $\delta_{f^{\prime}}(a A, b A) \geq 0$ for $a, b \in M x-A$. These inequalities follow easily from the listed conditions.

The identically zero function clearly satisfies these assumptions, thus it is an excess function. Actually, it adds a loop to the polymatroid. The inequality $\delta_{e}(A, B)+\delta_{f}(A, B) \geq 0$ holds for arbitrary subsets $A, B \subseteq M$ as this is just the modular defect of the pair $(A x, B x)$ in the extension.

The following statements connect one-point extensions, modular cuts and modular filters.

Proposition 3. The collection $\mathcal{G}$ of subsets of $M$ is a modular filter if and only if there is an excess function $e$ such that $e(M)=0$ and $\mathcal{G}=\{A \subseteq M: e(A)=0\}$.

Proof. If $e$ is an excess function with $e(M)=0$, then Proposition 2 and $\delta_{e}(A, B)+\delta_{f}(A, B) \geq 0$ trivially imply that $\mathcal{G}$ is a modular filter. To show the converse, let $\mathcal{G}$ be a modular filter, and choose the function $e$ as $e(A)=0$ for $A \in \mathcal{G}$, and $e(A)=\varepsilon$ otherwise where $\varepsilon$ is a sufficiently small positive value. We claim that conditions (i)-(iii) of Proposition 2 hold. This is clear for (i). For (ii) observe that $e(M)-e(M-i)$ is either 0 or $-\varepsilon$, and the latter holds when $M-i \notin \mathcal{G}$, but then $f(M-i) \neq f(M)$. Thus choosing $\varepsilon$ smaller than all positive $f(M)-f(M-i)$ ensures condition (ii).

Finally, $\delta_{e}(a A, b A)$ is either non-negative or equals $-\varepsilon$. This latter happens when both $a A$ and $b A$ are in $\mathcal{G}$ but $A \notin \mathcal{G}$. In this case $(a A, b A)$ is not a modular pair. Choosing $\varepsilon$ smaller than all possible positive modular defects in the polymatroid gives condition (iii).

\section{Claim 4. The following statements are equivalent:}

(i) The polymatroid $\mathcal{M}$ has a non-principal modular cut.

(ii) The polymatroid has flats $F_{1}$ and $F_{2}$ such that $F_{1} \cap F_{2} \notin$ $\mathcal{F}\left(F_{1}, F_{2}\right)$.

(iii) The polymatroid has flats $F_{1}$ and $F_{2}$ and an excess function e such that $e\left(F_{1}\right)=e\left(F_{2}\right)=0$ and $e\left(F_{1} \cap F_{2}\right)>0$.

Proof. (i) $\rightarrow$ (ii) If $\mathcal{F}$ is not a principal cut, then there are $F_{1}, F_{2} \in \mathcal{F}$ such that $S=F_{1} \cap F_{2} \notin \mathcal{F}$. As $\mathcal{F}\left(F_{1}, F_{2}\right)$ is a subcollection of $\mathcal{F}, S \notin \mathcal{F}\left(F_{1}, F_{2}\right)$.

(ii) $\rightarrow$ (iii) Let $\mathcal{F}=\mathcal{F}\left(F_{1}, F_{2}\right)$. By Proposition 1 $\mathcal{G}=$ $\mathrm{cl}^{-1}(\mathcal{F})$ is a modular filter, and by Proposition 3 there is an excess function $e$ such that $e(A)=0$ for $A \in \mathcal{G}$, and $e(A)>0$ otherwise. As $F_{1}, F_{2} \in \mathcal{G}$, the first required property holds, 
and the second also holds if we show that $S=F_{1} \cap F_{2} \notin \mathcal{G}$. But $S$ is a flat, $S \notin \mathcal{F}$, thus $S \notin \mathrm{cl}^{-1}(\mathcal{F})=\mathcal{G}$.

(iii) $\rightarrow$ (i) Let $e$ be the excess function, and consider the collection of flats $\mathcal{F}=\{F: e(F)=0\}$. Clearly, $F_{1}, F_{2} \in \mathcal{F}$ and $F_{1} \cap F_{2} \notin \mathcal{F}$. We claim that $\mathcal{F}$ is a modular cut. The fact that it is non-principal is clear. Property (i) and (iii) clearly hold. For (ii) observe that $\delta_{f}(A, B)+\delta_{e}(A, B) \geq 0$, thus if $A, B \in \mathcal{F}$, then $e(A)=e(B)=e(A \cup B)=0$ (as $e$ is decreasing and non-negative), and if $(A, B)$ is a modular pair, that is, $\delta_{f}(A, B)=0$, then

$$
0 \leq \delta_{f}(A, B)+\delta_{e}(A, B)=-e(A \cap B),
$$

meaning that $e(A \cap B)=0$, thus the flat $A \cap B$ is in $\mathcal{F}$.

\section{LINEAR POLYMATROIDS}

The polymatroid $\mathcal{M}=(f, M)$ is linearly representable if there is a (finite dimensional) vector space $V$ over some finite field and for each $i \in M$ a linear subspace $V_{i}$ of $V$ such that for all $A \subseteq M$, the rank of $A$ is the dimension of the subspace spanned by $V_{A}=\bigcup\left\{V_{i}: i \in A\right\}$; see [8].

A linearly representable polymatroid is clearly integer, and there are linearly representable polymatroids whose sum is not linearly representable. Frequently when linearly representable polymatroids have some interesting (or desired) property, so do polymatroids in their conic hull. The definition of linear polymatroids below illustrates such a case. As it captures one of most important aspect of linear representability, we hope that this notion has other applications.

Subsets $X, Y$ of the ground set are intersectable if either they form a modular pair, or there is an excess function $e$ such that $e(X)=e(Y)=0$ but $e(X \cap Y)>0$ or, equivalently, if $X \cap Y \notin \mathcal{G}(X, Y)$, see Proposition 3. The polymatroid is linear if every pair of its subsets is intersectable.

\section{Claim 5. Linearly representable polymatroids are linear.}

Proof. Suppose $\mathcal{M}=(f, M)$ is linearly representable; let $V_{i} \subseteq V$ be the linear subspace assigned to $i \in M$. For $A \subseteq M$, its rank is the dimension of the subspace spanned by $\bar{V}_{A}$. If the subsets $X$ and $Y$ are not modular, adjoin a new element to $\mathcal{M}$ represented by the intersection of the linear span of $V_{X}$ and the linear span of $V_{Y}$. Let $e$ be the excess function of this one-point extension. Then $e(X)=e(Y)=0$, and $e(X \cap Y)$ equals the modular defect of $X$ and $Y$, which is non-zero.

Claim 6. Conic combination of linear polymatroids is linear.

Proof. The definition of linear polymatroids is clearly invariant under multiplication. So suppose $\mathcal{M}_{1}$ and $\mathcal{M}_{2}$ are defined on the same ground set and both are linear. If $(X, Y)$ is modular in $\mathcal{M}_{i}$, then let $e_{i}$ be identically zero, otherwise let it be the excess function guaranteed by linearity. If $(X, Y)$ is not modular in $\mathcal{M}_{1}+\mathcal{M}_{2}$ then $e_{1}+e_{2}$ is the excess function showing the required extension.

Claim 7. Linear polymatroids satisfy (*).

Proof. Suppose $\left(F_{1}, F_{2}\right)$ is a non-modular pair of flats, we need to find a non-principal modular cut in the polymatroid. As the polymatroid is linear, there is an excess function $e$ with
$e\left(F_{1}\right)=e\left(F_{2}\right)=0$ and $e\left(F_{1} \cap F_{2}\right)>0$, and then the existence of non-principal modular cut follows from Claim 4.

\section{MAIN LEMMAS}

A non-negative linear (conic) combination of polymatroids on the same set $M$ is again a polymatroid on $M$. If $F$ is a flat in any constituent with positive coefficient, then $F$ is a flat in the sum; however the sum can have flats which are not flats in any of the constituents. The next lemmas establish properties of the constituents when their conic combination violates $(*)$.

Lemma 8. Let $\mathcal{M}$ and $\mathcal{N}$ be two polymatroids on the same set. Suppose $\mathcal{M}$ has two flats $F_{1}, F_{2}$ such that $F_{1} \cap F_{2} \notin$ $\mathcal{F}_{\mathcal{M}}\left(F_{1}, F_{2}\right)$. Then for any $\lambda>0, \mathcal{N}+\lambda \mathcal{M}$ satisfies $(*)$.

Proof. This holds since there is a non-principal modular cut in $\mathcal{N}+\lambda \mathcal{M}$. This follows from Claim 4 once we show that a) $F_{1}$ and $F_{2}$ are flats in $\mathcal{N}+\lambda \mathcal{M}$ (this is trivial from the discussion above), and $b$ ) there exists an appropriate excess function $e$ for $\mathcal{N}+\lambda \mathcal{M}$.

Let $e_{\mathcal{M}}$ be the excess function for $\mathcal{M}$ with $e_{\mathcal{M}}\left(F_{1}\right)=$ $e_{\mathcal{M}}\left(F_{2}\right)=0$, and $e_{\mathcal{M}}\left(F_{1} \cap F_{2}\right)>0$, guaranteed by the condition and Claim 4 and define $e=\lambda e_{\mathcal{M}}$. Conditions in Proposition 2 trivially hold (as they are linear), thus $e$ is the required excess function for $\mathcal{N}+\lambda \mathcal{M}$.

Lemma 9. Suppose $\lambda>0$ and $\mathcal{N}+\lambda \mathcal{M}$ is a minimal counterexample to $(*)$. In $\mathcal{M}$ every intersecting pair of flats is modular.

Proof. As $\mathcal{M}^{*}=\mathcal{N}+\lambda \mathcal{M}$ is a counterexample, it has a non-modular pair of flats but no non-principal modular cut. If $\left(F_{1}, F_{2}\right)$ is a non-modular pair of flats in $\mathcal{M}^{*}$ and $S=$ $F_{1} \cap F_{2}$ is not empty, then the contraction $M^{*} / S$ is a smaller counterexample to $(*)$. Consequently $\mathcal{M}^{*}$ has no intersecting non-modular flat pairs.

To finish the proof one has to notice that if $F_{1}$ and $F_{2}$ are intersecting non-modular flats in $\mathcal{M}$, then they remain the same in $\mathcal{M}^{*}$ as well.

From here the strategy for checking $(*)$ should be clear. Every polymatroid on a given ground set is a conic combination of finitely many extremal polymatroids which can be listed explicitly when the polymatroid has five or less elements [12]. Suppose $\mathcal{M}$ violates $(*)$ and no counterexample exists on a smaller ground set. This $\mathcal{M}$ is a conic combination of the extremal polymatroids. The combining coefficient is zero if the corresponding extremal polymatroid a) contains two intersecting non-modular flats (Lemma 9), or b) contains disjoint flats $F_{1}, F_{2}$ such that the modular cut $\mathcal{F}\left(F_{1}, F_{2}\right)$ is not principal (Lemma 8). This hopefully leaves only a few extremal polymatroids which can be checked individually.

\section{STICKY POLYMATROIDS ON FIVE OR FEWER ELEMENTS}

\section{A. Polymatroids on two elements}

Let $M=\{a, b\}$. Polymatroids on $M$ are conic combinations of the three extremal ones listed in Table ! Each of them is linearly representable, thus linear. By Claim 6 their conic combination remains linear. Thus every polymatroid on two elements is linear, and by Claim 7 they satisfy $(*)$. 


$\begin{array}{lrrr} & a & b & a b \\ \mathcal{M}_{a} & 1 & 0 & 1 \\ \mathcal{M}_{b} & 0 & 1 & 1 \\ \mathcal{M}_{a b} & 1 & 1 & 1\end{array}$

EXTREMAL POLYMATROIDS ON $\{a, b\}$

\section{B. Polymatroids on three elements}

There are eight extremal polymatroids on $M=\{a, b, c\}$. Up to isomorphism there are four different ones listed in Table I the others can be obtained by permuting the elements of $M$. As

\begin{tabular}{llllcccc} 
& $a$ & $b$ & $c$ & $a b$ & $a c$ & $b c$ & $a b c$ \\
$\mathcal{M}_{a}$ & 1 & 0 & 0 & 1 & 1 & 0 & 1 \\
$\mathcal{M}_{a b}$ & 1 & 1 & 0 & 1 & 1 & 1 & 1 \\
$\mathcal{M}_{a b c}$ & 1 & 1 & 1 & 1 & 1 & 1 & 1 \\
$\mathcal{M}_{*}$ & 1 & 1 & 1 & 2 & 2 & 2 & 2 \\
\multicolumn{7}{c}{ TABLE II } \\
EXTREMAL POLYMATROIDS ON $\{a, b, c\}$
\end{tabular}

in the two-element case, all of them are linearly representable, thus every polymatroid on $\{a, b, c\}$ is linear. By Claim 7, (*) holds for these polymatroids. In $\mathcal{M}_{*}$, every pair of singletons is independent (modular), but any two determine the third one.

\section{Polymatroids on four elements}

Extremal polymatroids on four and five elements have been reported in [12]. The software package Polco [11] can generate the extremal rays from the collection of the defining inequalities. The polymatroid cone $\Gamma_{a b c d}$ has 41 extremal rays. There are only 11 different among the corresponding polymatroids up to isomorphism. Table III lists one element from each isomorphism class; the ranks are shown as follows: first oneelement subsets, then two-element subsets, etc., each group in alphabetical order. Polymatroids $\mathcal{M}_{1}-\mathcal{M}_{10}$ are linearly

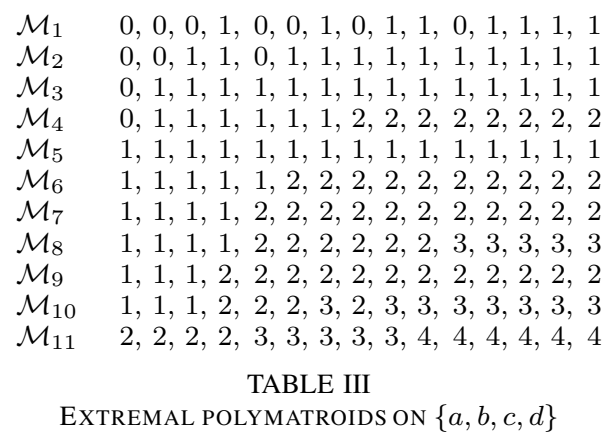

representable. For $\mathcal{M}_{10}$ take three linearly independent vectors $\mathbf{a}, \mathbf{b}, \mathbf{c}$; they span the one-dimensional subspaces assigned to $a, b, c$, respectively, while $d$ gets the 2-dimensional subspace spanned by $\mathbf{a}+\mathbf{b}$ and $\mathbf{a}+\mathbf{c}$. The polymatroid $\mathcal{M}_{11}$ is not linearly representable, but the modular cut generated by the flats $a c$ and $b d$ is $\{a c, b d, a b c d\}$. Thus by Lemma 8 it cannot contribute to a $(*)$-violating polymatroid. Consequently each polymatroid on four elements satisfies $(*)$.

\section{Polymatroids on five elements}

According to [12] there are 117983 extremal polymatroids on a five element set. Up to isomorphism there are 1320 different ones. By Lemmas 8 and 9 we can eliminate those extremal polymatroids that a) contain two intersecting nonmodular flats, or b) contain disjoint flats $F_{1}, F_{2}$ such that $\mathcal{F}\left(F_{1}, F_{2}\right)$ is not principal (i.e., the generated cut does not contain the empty set). After this thinning we get a quite
$0,0,0,0,1,0,0,0,1,0,0,1,0,1,1,0,0,1,0,1,1,0,1,1,1,0,1,1,1,1,1$ $0,0,0,1,1,0,0,1,1,0,1,1,1,1,1,0,1,1,1,1,1,1,1,1,1,1,1,1,1,1,1$ $0,0,1,1,1,0,1,1,1,1,1,1,1,1,1,1,1,1,1,1,1,1,1,1,1,1,1,1,1,1,1$ $0,0,1,1,1,0,1,1,1,1,1,1,2,2,2,1,1,1,2,2,2,2,2,2,2,2,2,2,2,2,2$ $0,1,1,1,1,1,1,1,1,1,1,1,1,1,1,1,1,1,1,1,1,1,1,1,1,1,1,1,1,1,1$ $0,1,1,1,1,1,1,1,1,1,2,2,2,2,2,1,2,2,2,2,2,2,2,2,2,2,2,2,2,2,2$ $0,1,1,1,1,1,1,1,1,2,2,2,2,2,2,2,2,2,2,2,2,2,2,2,2,2,2,2,2,2,2$ $0,1,1,1,2,1,1,1,2,2,2,2,2,2,2,2,2,2,2,2,2,2,2,2,2,2,2,2,2,2,2$ $1,1,1,1,1,1,1,1,1,1,1,1,1,1,1,1,1,1,1,1,1,1,1,1,1,1,1,1,1,1,1$ $1,1,1,1,1,1,1,2,2,1,2,2,2,2,2,1,2,2,2,2,2,2,2,2,2,2,2,2,2,2,2$ $1,1,1,1,1,1,2,2,2,2,2,2,1,2,2,2,2,2,2,2,2,2,2,2,2,2,2,2,2,2,2$ $1,1,1,1,1,1,2,2,2,2,2,2,2,2,2,2,2,2,2,2,2,2,2,2,2,2,2,2,2,2,2$ $1,1,1,1,1,2,2,2,2,2,2,2,2,2,2,2,2,2,2,2,2,2,2,2,2,2,2,2,2,2,2$ $1,1,1,1,2,1,2,2,2,2,2,2,2,2,2,2,2,2,2,2,2,2,2,2,2,2,2,2,2,2,2$ $1,1,1,1,2,2,2,2,2,2,2,2,2,2,2,2,2,2,2,2,2,2,2,2,2,2,2,2,2,2,2$ $1,1,1,2,2,2,2,2,2,2,2,2,2,2,2,2,2,2,2,2,2,2,2,2,2,2,2,2,2,2,2$ $1,1,1,2,2,2,2,2,2,2,3,3,3,3,3,2,3,3,3,3,3,3,3,3,3,3,3,3,3,3,3$

\section{TABLE IV}

REMAINING EXTREMAL POLYMATROIDS ON $\{a, b, c, d, e\}$

meager set of 17 isomorphism classes; representatives are listed in Table IV The ranks are shown by cardinality of the subset, and within that alphabetically. By inspection, all of them are linearly representable, thus linear. By Claim 6 , any conic combination of linear polymatroids is linear, and Claim 7 says that these linear polymatroids satisfy $(*)$. Consequently all polymatroids on 5 elements satisfy $(*)$.

\section{ACKNOWLEDGMENT}

The research reported in this paper was supported by GACR project number 19-04579S, and partially by the Lendület program of the HAS.

\section{REFERENCES}

[1] A. Bachem and W. Kern (1988) On sticky matroids, Discrete Math 69 $11-18$

[2] J. E. Bonin (2011) A note on the sticky matroid conjecture, Ann. Comb. 15 619-624

[3] L. Csirmaz (2019), Sticky matroids and convolution. Available at arXiv.org, arXiv:1909.02353

[4] R. Dougherty, C. Freiling, K. Zeger (2009) Linear rank inequalities on five or more variables. Available at arXiv.org, arXiv:0910.0284

[5] W. Hochstättler, M. Wilhelmi (2019) Sticky matroids and Kantor's conjecture, Algebra Univers. 80-12, pp. 1-21

[6] L. Lovász (1982) Submodular functions and convexity. Mathematical Programming - The State of the Art (A. Bachem, M. Grötchel and B. Korte, eds.), Springer-Verlag, Berlin, 234-257.

[7] F. Matúš (2007) Adhesivity of polymatroids, Discrete Mathematics 307 pp. 2464-2477

[8] J.G. Oxley (1992) Matroid Theory, Oxford Science Publications. The Calrendon Press, Oxford University Press, New York

[9] C. Padro (2012) Lecture Notes in Secret Sharing, Cryptology ePrint Archive 2012/674

[10] S. Poljak, D. Turzik (1982) A note on sticky matroids, Discrete Math, 42(1) 119-123

[11] M. Terzer (2009) Polco: A Java tool to compute extreme rays of polyhedral cones. Available at http:// www.csb.ethz.ch/tools/ polco

[12] M. Studeny, R. R. Bouckaert, T. Kocka (2000) Extreme supermodular set functions over five variables Research Report no 1977, Institute of Information Theory and Automation, Prague

[13] R. W. Yeung (2002) A first course in information theory, (2002) Kluwer Academic / Plenum Publishers 